\title{
Coarse-grained modeling study of nonpeptide RGD ligand density and PEG molecular weight on the conformation of poly $(\gamma$-glutamyl-glutamate) paclitaxel conjugates
}

\author{
Lili X. Peng • Sanjib K. Das • Lei Yu • \\ Stephen B. Howell • David A. Gough
}

Received: 11 October 2010 / Accepted: 24 January 2011 /Published online: 1 March 2011

(C) The Author(s) 2011. This article is published with open access at Springerlink.com

\begin{abstract}
Molecular shape, flexibility, and surface hydrophilicity are thought to influence the ability of nanoparticles to cross biological barriers during drug delivery. In this study, coarse-grained (CG) molecular dynamics (MD) simulations were used to study these properties of a polymer-drug construct in potential clinical development: poly( $\gamma$-glutamyl-glutamate)-paclitaxel-poly(ethylene glycol) nonpeptide RGD (PGG-PTX-PEG-npRGD), a linear glutamyl-glutamate polymer with paclitaxel and poly(ethylene glycol)-nonpeptide RGD side groups. It was hypothesized that the PEG molecular weight (MW) (500 Da; 1,000 Da; and 2,000 Da) and nonpeptide RGD ligand density (4, 8, 12, and 16 per molecule), respectively, may have advantageous effects on the shape, flexibility, and surface hydrophilicity of PGG-PTX-PEG-npRGD. Circular dichroism spectroscopy was used to suggest initial structures for the all-atom (AA) models of PGG-PTX-PEGnpRGD, which were further converted to CG models using
\end{abstract}

Electronic supplementary material The online version of this article (doi:10.1007/s00894-011-0989-4) contains supplementary material, which is available to authorized users.

L. X. Peng $\cdot$ D. A. Gough $(\bowtie)$

Department of Bioengineering, University of California,

San Diego,

La Jolla, CA, USA

e-mail: dgough@ucsd.edu

L. X. Peng

e-mail: lilipeng@gmail.com

S. K. Das $\cdot$ L. Yu

Nitto Denko Technical Corporation,

Oceanside, CA, USA

S. B. Howell

Moores Cancer Center, University of California, San Diego,

La Jolla, CA, USA a commercially available mapping algorithm. Due to its semi-flexibility, PGG-PTX-PEG-npRGD is not limited to one specific conformation. Thus, CG MD simulations were run until statistical equilibrium, at which PGG-PTX-PEG$n p R G D$ is represented as an ensemble of statistically similar conformations. The size of a PGG-PTX-PEG-npRGD molecule is not affected by the PEG MW or the nonpeptide RGD density, but higher PEG MW results in increased surface density of a PGG-PTX-PEG-npRGD molecule. Most PGG-PTX-PEG-npRGD shapes are globular, although filamentous shapes were also observed in the PEG500 and PEG1000 molecules. PEG500 and PEG1000 molecules are more flexible than PEG2000 systems. A higher presence of npRGD ligands results in decrease surface hydrophilicity of PGG-PTX-PEG-npRGD. These results indicate that the PGG-PTX-PEG1000-npRGD 4 and PGG-PTX-PEG1000-npRGD 8 molecules are the most efficacious candidates and are further recommended for experimental preclinical studies.

Keywords Polymer Paclitaxel · Poly(ethylene glycol) . Nonpeptide RGD · Active targeting · Coarse-grained modeling

\section{Introduction}

A continuing challenge in the development of anticancer therapeutics is ensuring that adequate amounts of the drug are delivered to tumors while simultaneously minimizing toxic and adverse effects to healthy tissue [1, 2]. As angiogenesis is crucial for tumor growth, the microvascular endothelial cell and its receptors have become key target modulators in cancer therapy [3]. The $\alpha_{V} \beta_{3}$ integrin and its ligands, vitronectin and fibronectin, are known to promote 
angiogenesis [4, 5], and tumor cells with non-activated $\alpha_{V} \beta_{3}$ integrins have been shown to exhibit decreased angiogenic behavior [6]. As a result, antagonists of $\alpha_{V} \beta_{3}$ integrins have emerged as a new class of anticancer therapeutics, primarily the Arg-Gly-Asp (RGD) tripeptide and RGD peptidomimetics, which bind to $\alpha_{V} \beta_{3}$ integrins with high affinity to prevent binding of $\alpha_{V} \beta_{3}$ integrins to their natural receptors [7-9].

This study focuses on the preclinical development of a nonpeptide RGD (npRGD)-based drug delivery system: poly $(\gamma$-glutamyl-glutamate) paclitaxel tethered to npRGD via poly(ethylene glycol) (PGG-PTX-PEG-npRGD) [10]. Paclitaxel (Taxol ${ }^{\circledR}, \mathrm{C}_{47} \mathrm{H}_{51} \mathrm{NO}_{14}$ ) (PTX) is a hydrophobic drug used to treat breast, ovarian, and lung cancers, and poly $(\gamma$-glutamyl-glutamate) (PGG) is a hydrophilic polymer conjugated to PTX to improve solubility [11, 12]. To maximize access of npRGD to $\alpha_{V} \beta_{3}$ integrins, PEG is positioned between glutamyl-glutamate and npRGD residues to avoid steric interactions between PGG-PTX and npRGD (see Fig. 1). Conjugation of PEG to therapeutic agents has also been shown to result in improved in vivo stability, protection from proteolytic digestion, increased biological half-life, improved solubility, and decreased toxicity [13].

Preclinical development of anticancer therapeutics usually involves trial-and-error testing of candidate compounds on in vitro and in vivo models. While these methods yield useful results, they are time-consuming, inefficient, and resource-intensive. Given to urgent clinical need for successful therapies there is clear motivation to shorten the course of preclinical development of therapeutic agents. In this study, we performed molecular dynamics (MD) simulations of PGG-PTX to predict their physicochemical properties, such as shape, flexibility, and surface hydro-
Fig. 1 Chemical structures of poly $(\gamma$-glutamyl-glutamate) (GG), GG-paclitaxel (PTX), and GG-poly(ethylene glycol) (PEG)-npRGD [non-peptide residues RGD (Arg-Gly-Asp) peptidomimic]. $\mathrm{GG}_{\mathrm{NH} 3+}$ and GG-PTX each has a charge of -1 ; GG and GG-PEG-npRGD each a charge of -2 ; and $\mathrm{GG}_{\mathrm{COO}-}$ a charge of -3 . The GG residue differs slightly based on its position on the polymer, e.g., its position at the amino or carboxyl termini. For the GG-PTX residue, paclitaxel is conjugated to a carboxylate group of glutamyl-glutamate via an ester linkage. For the GGnpRGD residue, the nonpeptide RGD is attached to a linear PEG spacer, which is conjugated to the GG residue. The length $n$ of the PEG spacer depends on the molecular weight of the linear PEG molecule: $500 \mathrm{Da} ; 1,000$ $\mathrm{Da}$; and 2,000 Da correspond to approximately 11,23 , and 45 ethylene glycol monomers

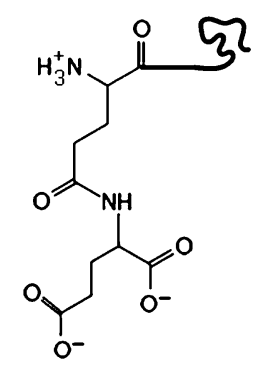

Glutam yl-glutamate, $\mathrm{NH}_{3}^{+}$terminus ( $\left(\mathrm{G}_{\mathrm{NH} 3+}\right)$

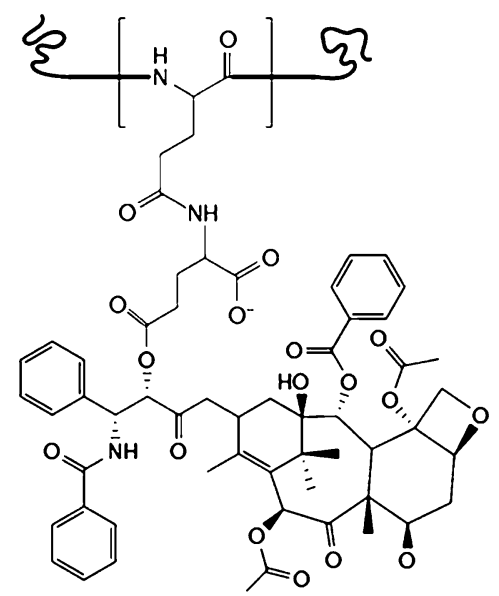

Glutamyl-glutamate - paclitaxel (GG-PTX)<smiles>CC(C)(C)NC(CCC(=O)NC(CCC(O)O)C(=O)[O-])C(=O)C(C)(C)C(C)(C)C</smiles>

Glutamyl-glutamate (GG)
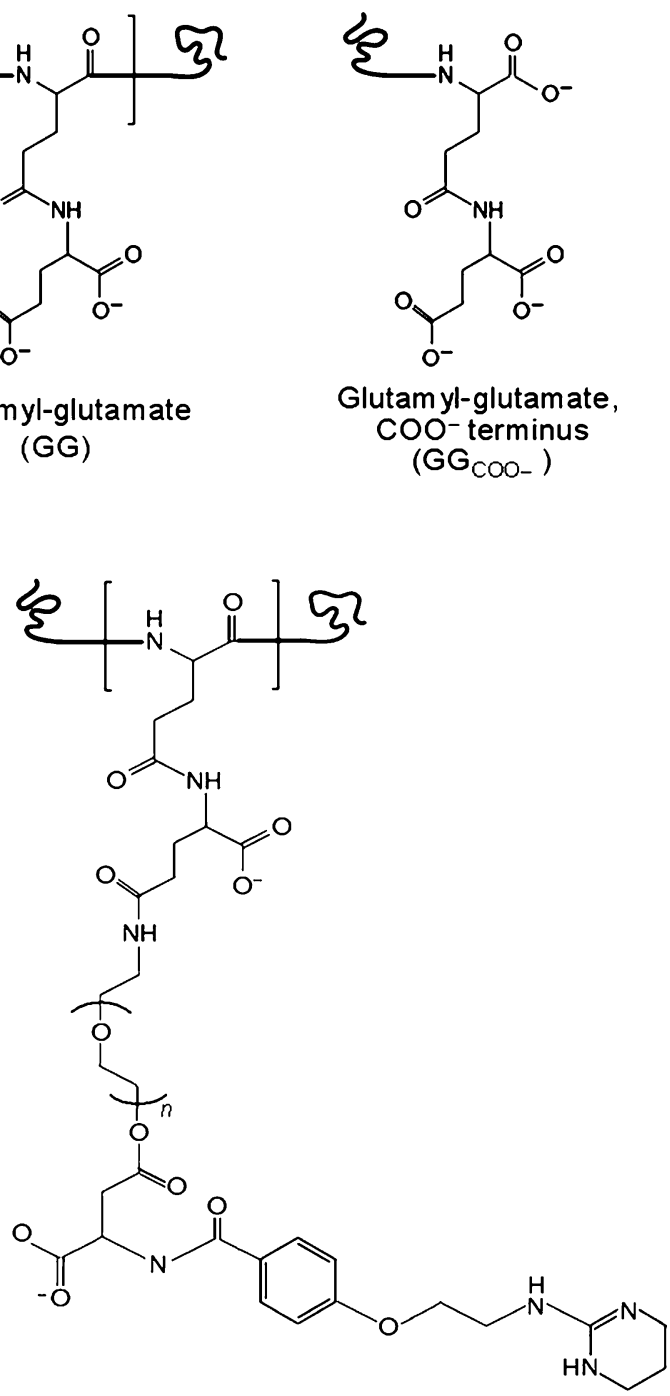

Glutamyl-glutamate - poly(ethylene glycol) $)_{n}$ - nonpeptide RGD (GG-PEG $\left.{ }_{n}-\mathrm{npRGD}\right)$ 
philicity-factors that may influence how nanoparticles overcome biological barriers [14-18]. It was hypothesized that the density of nonpeptide RGD $(4,8,12$, and 16 ligands) and molecular weight (MW) of PEG spacer (500 $\mathrm{Da}$; 1,000 Da; and 2,000 Da) concomitantly affects the shape, flexibility, and surface hydrophilicity of a PGGPTX-PEG-npRGD molecule. (Figure 2 shows the patterning schemes for the PGG-PTX-PEG-npRGD molecules.)
Theoretical insight from the modeling results may be useful to suggest better molecular designs of PGGPTX-PEG-npRGD, ultimately expediting its preclinical development.

Prior to running MD simulations, a key challenge was to determine the initial configuration of PGG-PTX-PEGnpRGD [19]. The combination of flexible polymeric components (PGG and PEG) and rigid components (PTX

\section{4 nonpeptide RGD}

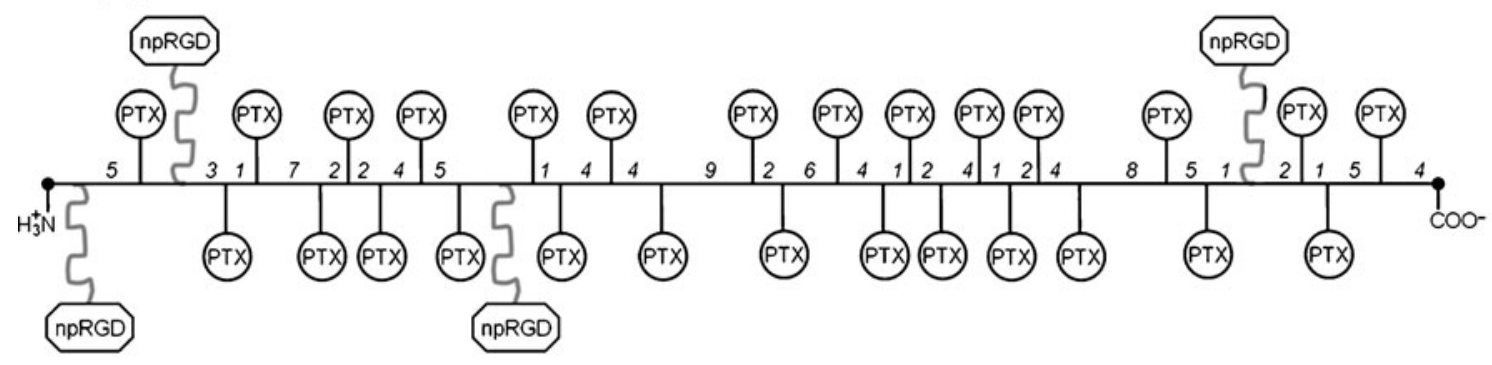

8 nonpeptide RGD

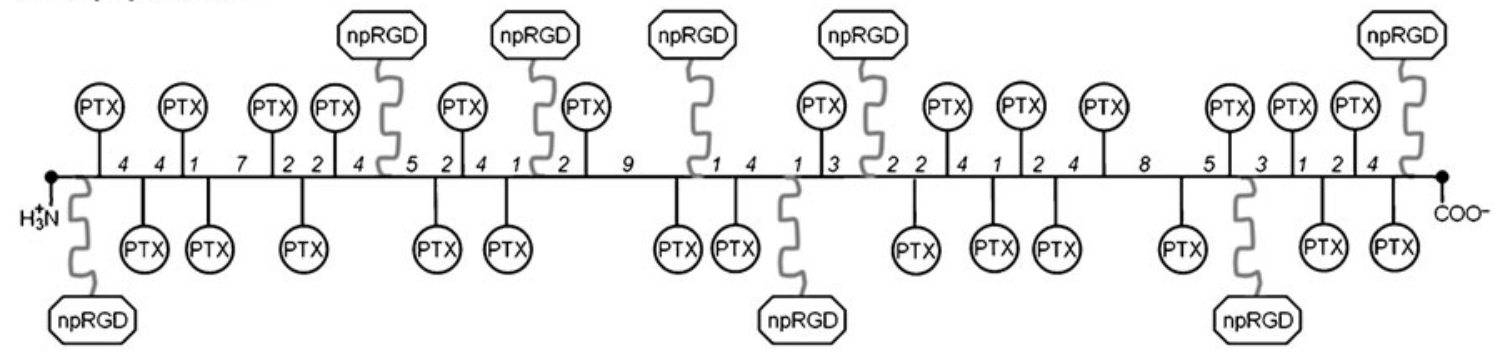

12 nonpeptide RGD

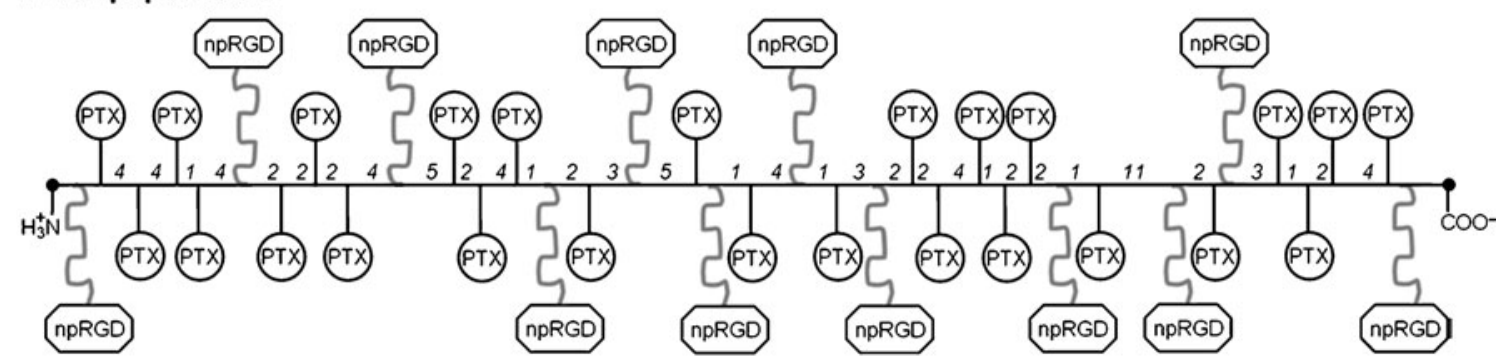

16 nonpeptide RGD

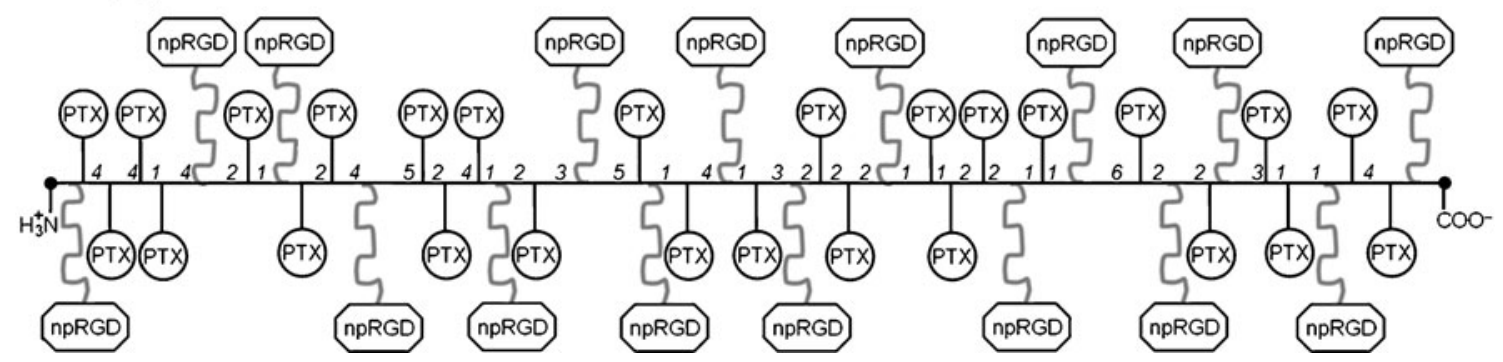

Fig. 2 Abstract representations of the spatial positioning patterns of paclitaxel (PTX) and (PEG) ${ }_{n}$-nonpeptide RGD molecules on the PGG backbone. Patterning schemes apply to all PEG 500, PEG 1000, and PEG 2000 systems. Each PGG-PTX molecule is composed of 130 poly- $\gamma$-glutamyl-glutamate monomers, 26 paclitaxel molecules, and 4 , 8,12 , or $16(\mathrm{PEG})_{n}$-nonpeptide RGD molecules. Paclitaxel and
(PEG) ${ }_{n}$-nonpeptide RGD molecules are attached covalently to the PGG backbone in a random fashion. Numbers between residues denote the number of repeating GG residues that are not amino- or carboxyl-termini GG residues. The amino- and carboxyl-termini GG residues are represented by black dots at the ends of each line 
and npRGD) make PGG-PTX-PEG-npRGD a semiflexible molecule. Currently, there is no crystallized conformation representing its experimental form available from the Protein Data Bank. Therefore, circular dichroism (CD) spectroscopy was used to determine the presence of chiral forms of PGG-PTX-PEG-npRGD in aqueous form and obtain a general idea of its initial structure, which was then used to construct all-atom (AA) models.

In previous work on protein simulations, once the initial configuration has been determined, MD simulations were run to equilibrium, at which point a specific structure is attained $[20,21]$. At this point, the potential energy level of a system became constant with respect to time. The approach must be different in the present case. Given the semiflexible nature of the PGG-PTX-PEG-npRGD, the molecule most likely exists as a population of related structures, rather than one specific structure. Therefore, the goal is not to attain a static PGGPTX-PEG-npRGD conformation through MD simulations. Rather, MD simulations are run until minimal structural fluctuations at the molecular level, or a statistical equilibrium [22], is reached, at which point a PGG-PTX-PEG-npRGD molecule is characterized as an ensemble of statistically similar structures.

Given the relatively large sizes of each PGG-PTX-PEGnpRGD molecule ( 90-130 kDa), running MD simulations on AA models in explicit solvent was judged to require an extraordinary amount of CPU time and expense. Therefore, to minimize computational costs and allow access longer time scales, AA models were converted to coarse-grained (CG) models using the mapping scheme in the MARrink's Toolkit INItiative (MARTINI) force field [23]. CG parameterization of the models was done by using the Boltzmann inversion to determine the equilibrium bonded distances and angles from $100 \mathrm{~ns}$ AA MD simulations in implicit solvent.

\section{Experimental and computational methods}

\section{Experimental}

\section{Sample preparation}

A lyophilized sample of PGG-PTX-PEG220-npRGD 16 was provided by Nitto Denko Technical Corporation (Oceanside, CA, USA). The sample was weighed and then diluted to $1 \mathrm{mg} / \mathrm{ml}$ in $1 \mathrm{X}$ HyClone modified DPBS buffer (Cat. No. SH30028.03, ThermoScientific, Fremont, CA). The sample was then sonicated in a $37^{\circ} \mathrm{C}$ water bath for $15 \mathrm{~min}$ and allowed to settle at room temperature $\left(25^{\circ} \mathrm{C}\right)$ for $10 \mathrm{~min}$. Finally, the sample was filtered using a $0.2 \mu \mathrm{m}$ filter paper (Part No. 431215, Corning Life Sciences, San Francisco, CA, USA) and a 20G 1 1/2 Precision Glide needle (Becton Dickinson, Franklin Lakes, NJ, USA).

\section{Circular dichroism spectroscopy}

CD spectroscopy measurements of the PGG-PTXPEG220-npRGD 16 sample were carried out using an AVIV Model 202 spectrophotometer (AVIV Biomedical, Lakewood, NJ, USA). CD spectra of pure 1X DPBS buffer and FD protein in $50 \%$ TFE $/ 50 \%$ double distilled $\mathrm{H}_{2} \mathrm{O} / 0.1 \%$ TFA were also taken as the negative and positive controls, respectively. All measurements were taken at $37^{\circ} \mathrm{C}$. The far-UV CD spectra were recorded from 190 to $260 \mathrm{~nm}$ using a $1-\mathrm{cm}$ rectangular quartz cuvette, and the $\mathrm{CD}$ spectra were collected at every $0.5 \mathrm{~nm}$ with $5 \mathrm{sec}$ at each point. For each sample except pure 1X DPBS, the concentration was adjusted and diluted so that the dynode voltage corresponding to the ellipticity signal remained below $500 \mathrm{~V}$ throughout the entire spectra collection period. (It has been suggested in Greenfield et al. [24] that the signalto-noise ratio diminishes greatly once the dynode voltage exceeds 500 V.) The optimum concentrations of each sample leading to the optimal elliptical signal and lowest signal-to-noise ratio were determined to be: $1 \mathrm{mg} / \mathrm{ml}$ of PGG-PTX-PEG220-npRGD 16 in $1 X$ DPBS and $0.25 \mathrm{mg} / \mathrm{ml}$ of FD protein in $50 \%$ TFE $/ 50 \% \mathrm{ddH}_{2} \mathrm{O} / 0.1 \%$ TFA.

Computational methods

\section{All-atom modeling}

Model construction The initial structures and input files for the following residues were constructed in the xleap module of AMBER 9.0: $\mathrm{GG}, \mathrm{GG}_{\mathrm{COO}-}, \mathrm{GG}_{\mathrm{NH} 3+}$, GGPTX, GG-PEG500-npRGD, GG-PEG1000-npRGD, and GG-PEG2000-npRGD. The initial structure for paclitaxel (PDB ID: 1JFF) was provided by the Protein Data Bank [25]. The Gaussian (g03) program's geometry optimization and restrained electrostatic potential fitting (RESP) were used to generate the atom-centered point charges for all residues $[26,27]$. For the protonated molecules, hydrogen atoms were added to the carboxyl groups to achieve their ionized states. To obtain the electrostatic potential for these residues, the AM1 geometry scheme and the $\mathrm{HF} / 6-31 \mathrm{G}^{*}$ and $\mathrm{HF} / 6-31 \mathrm{G}^{* *}$ pop $=\mathrm{mk} \operatorname{iop}(6 / 33=2) \mathrm{ab}$ initio level calculations were performed using the Gaussian program. Finally, to derive the equivalent partial atomic charges, RESP fitting was applied on the electrostatic potentials.

Energy minimization and MD simulation These steps were carried out using the AMBER 9.0 sander module. Each molecule was solvated and minimized in implicit solvent using the modified Generalized-Born model of IGB $=2$ and the linear combinations of pairwise overlaps (LPCO) model $[28,29] ; 250$ steps of steepest descent followed by 1,750 steps of conjugate gradient were carried out. The ionic 
strength of the implicit solvent was set to $140 \mathrm{mM}$ to mimic the salt concentration of blood plasma. No periodic boundary conditions were applied. A non-bonded electrostatic cutoff of $16.0 \AA$ was used. Trajectory snapshots were saved every 100 steps for further reprocessing.

MD simulations were also carried out using the sander module applying the modified version (ff99SB) of the Cornell et al. parm99 and GAFF force fields in the NVT ensemble (in which the number of atoms $\mathrm{N}$, volume $\mathrm{V}$, and temperature $\mathrm{T}$ were fixed) at $310 \mathrm{~K}$ in implicit solvent without periodic boundary conditions [30-32]. Constant temperature scaling was also applied with a time constant of 0.5 ps. Langevin dynamics was used with a collision frequency of $2.0 \mathrm{ps}^{-1}$. Newton's equations of motions were integrated with a time step of 2 fs. All bonds involving hydrogen atoms were constrained using the SHAKE algorithm [33]. The rotational and translational degrees of freedom about the center of mass were eliminated. Trajectory snapshots were saved every 100 steps for later reprocessing. Each cycle of the MD simulation was run for $0.1 \mathrm{~ns}$; this step was repeated until a statistical equilibrium was reached at $100 \mathrm{~ns}$.

\section{Coarse-grained modeling}

CG mapping The MARTINI force field was selected for the CG parameterization for its successful application to proteins by experimental validation of their structural and thermodynamic properties as well as the amino acid-based nature of the glutamyl-glutamate backbone [23, 34]. The MARTINI force field dictates that a group of roughly fourfive atoms are represented as an interaction center, or bead. The only exception applies to aromatic groups, in which a mapping scheme of two heavy atoms-to-one-bead was applied in order to maintain geometric symmetry, namely, the benzene entities in paclitaxel and nonpeptide RGD. These beads interact through a set of short-range Lennard-Jones potentials to reproduce characteristic properties resulting from AA simulations. Charged groups interact via a Coulombic energy function, and bonded potentials are used to describe the chemical connectivity of the beads. CG mapping of the $\mathrm{GG}, \mathrm{GG}_{\mathrm{COO}-}, \mathrm{GG}_{\mathrm{NH} 3+}$, and GG-PTX residues was previously done in Peng et al. [22]. CG mapping of the GG-PEG500npRGD residue is shown in Fig. 3, and CG mapping of the GG-PEG1000-npRGD and GG-PEG2000-npRGD residues are provided in Fig. S2. Also, each $\mathrm{Na}+$ ion is represented as a single $\mathrm{Q}$ type bead, and four AA water molecules are mapped to a single $\mathrm{P}_{4}$ type bead (W).

\section{Theory}

The dynamics of the beads are described by Newton's equations of motion. The effective bonded and nonbonded interactions are described by a potential of the form, adapted from Marrink et al. [34]:

$V=\sum_{m}\left[V_{\text {bond }}^{m}+V_{\text {angle }}^{m}+V_{\text {dihedral }}^{m}\right]+V_{\text {nonbonded }}^{m}$

where the index $m$ represents a bead from a PGG-PTX-PEGnpRGD molecule, a sodium $(\mathrm{Na}+)$ ion, or a water (W) bead. $V_{\text {bond }}$ represents the forces between two successively bonded beads, $V_{\text {bond }}$ accounts for the forces used to sustain angles between three successively bonded beads, and $V_{\text {dihedral }}$ corresponds to the dihedral angle potential for four successively bonded beads. The $V_{\text {nonbonded }}$ term accounts for the nonbonded interactions among all of the beads in a system. The bonded interactions are used to describe the chemical connectivity of the beads and are characterized as one of the following (the indices $i$ and $j$ represent two consecutive beads):

$V_{b o n d}^{m}=\sum_{i j} \frac{1}{2} k_{i j}^{m}\left(r_{i j}^{m}-r_{0, i j}^{m}\right)^{2}$

where $r_{i j}^{m}$ is the distance between two consecutive beads $i$ and $j$, $r_{0, i j}^{m}$ is the equilibrium bond length, and $k_{i j}^{m}$ is the distance force constant. The potential for bond angles is:

$V_{\text {angle }}^{m}=\sum_{i j k} \frac{1}{2} k_{i j k}^{m}\left[\cos \left(\theta_{i j k}^{m}\right)-\cos \left(\theta_{0, i j k}^{m}\right)\right]^{2}$

where $\theta_{i j k}^{m}$ is the angle between beads $i, j$, and $k, \theta_{0, j i k}^{m}$ is the equilibrium angle, and $k_{i j k}^{m}$ is the angle force constant.

The dihedral potential, $V_{\text {dihedral }}$, applies only to backbone protein residues. Since the general structure of PGG-PTXPEG-npRGD adapts a random coil, $V_{\text {dihedral }}$ was set to 0 for all combinations of four successively connected beads:

$V_{\text {dihedral }}^{m}=\sum_{i j k l} k_{i j k l}^{m}\left[1+\cos \left(n \chi_{i j k l}^{m}-\delta_{i j k l}^{m}\right)\right]$

where $n$ is an integer that represents multiplicity, $\delta_{i j k l}^{m}$ is the phase shift, $\chi_{i j k l}^{m}$ is the angle between the plane formed by the first three beads $i, j$, and $k$ and the plane formed by the last three beads $j, k$, and $l$, and $k_{i j k l}^{m}$ is the dihedral force constant.

Nonbonded interactions between two beads are described by a van der Waals (vdW) potential, characterized by a Lennard-Jones 12-6 potential energy function and a Coulombic potential for charged beads. The following summation is performed over all pairs of nonbonded beads $m$ and $n$ separated by a distance of $r_{\mathrm{mn}}$ :

$$
\begin{aligned}
V_{\text {nonbonded }}= & \sum_{m, n} 4 \varepsilon_{m n}\left[\left(\frac{\sigma_{m n}}{r_{m n}}\right)^{12}-\left(\frac{\sigma_{m n}}{r_{m n}}\right)^{6}\right] \\
& +\sum_{m, n} \frac{q_{m} q_{n}}{4 \pi \varepsilon_{0} \varepsilon r_{m n}}
\end{aligned}
$$

where constants $\sigma_{\mathrm{mn}}$ and $\varepsilon_{\mathrm{mn}}$ are the vdW parameters for the bead interaction, $\sigma_{\mathrm{mn}}$ refers to the closest distance of 


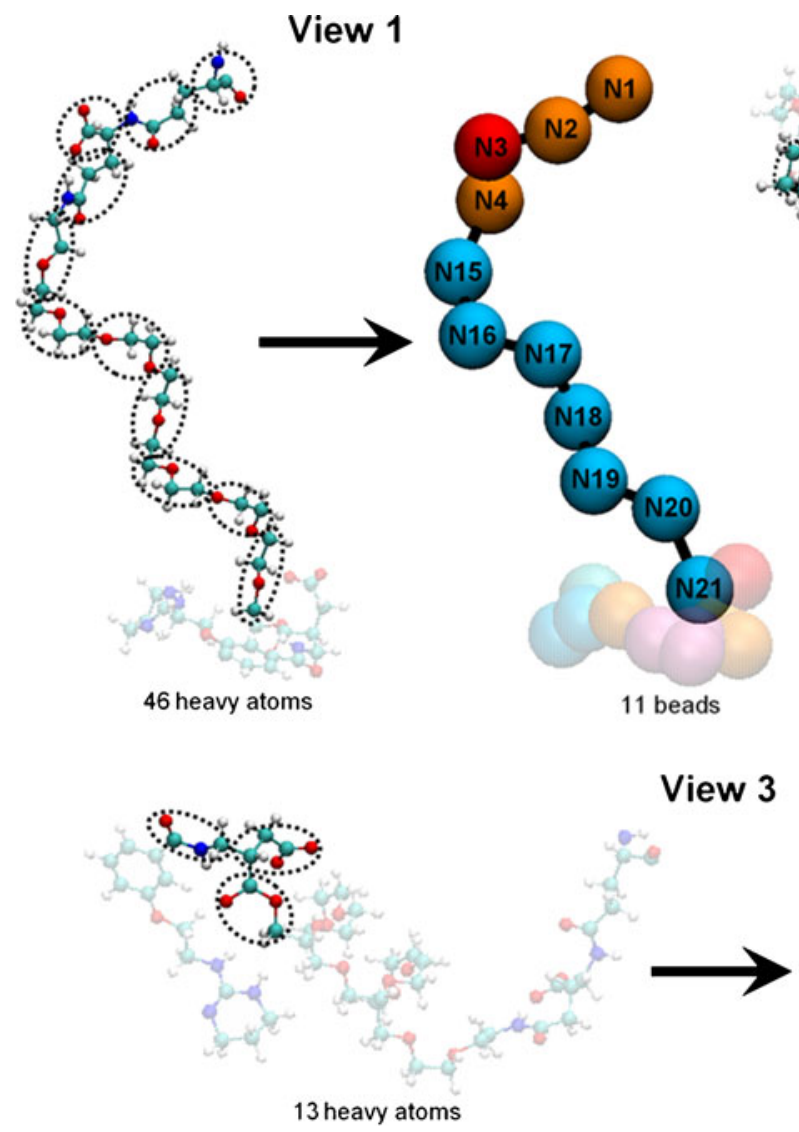

View 2

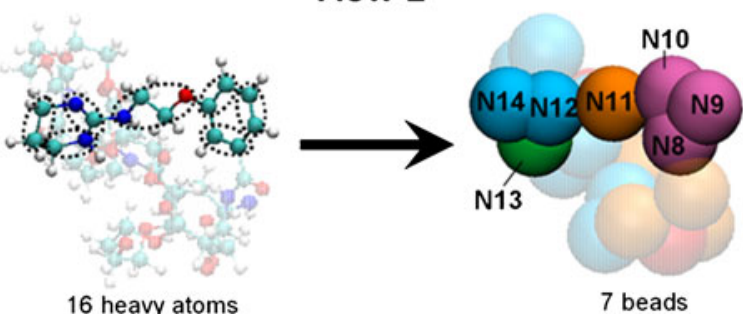

16 heavy atoms

7 beads

\begin{tabular}{|lll|}
\hline \multicolumn{3}{c|}{ Corresponding Bead Types } \\
N1: BP5 & N8: SC4 & N15: N0 \\
N2: P4 & N9: SC4 & N16: N0 \\
N3: Qa & N10: SC4 & N17: N0 \\
N4: P4 & N11: P3 & N18: N0 \\
N5: P3 & N12: SNa & N19: N0 \\
N6: Qa & N13: SC1 & N20: N0 \\
N7: P3 & N14:SNd & N21: N0 \\
\multicolumn{4}{|c}{ Range of Polarity } \\
apolar & intermediate polar charged \\
\hline
\end{tabular}

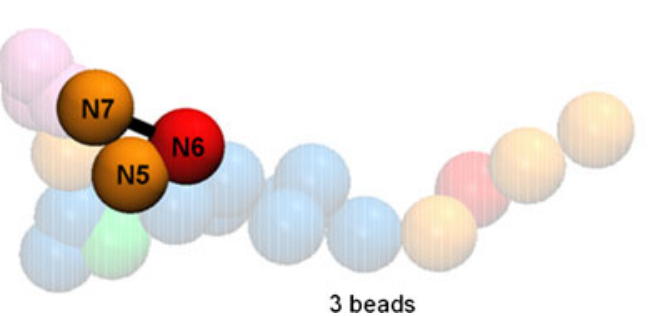

Fig. 3 Coarse-grained representation of a GG-PEG500-npRGD residue. A GG-PEG500-npRGD molecule was reduced from 75 heavy atoms to 21 CG beads. (CG representations for the GG-PEG1000-npRGD and GG-PEG2000-npRGD residues are shown in Figs. S1 and S2, respectively)

approach between two beads $m$ and $n$, and $\varepsilon_{\mathrm{mn}}$ denotes the strength of the interactions between beads $m$ and $n$. The charges $q_{\mathrm{m}}$ and $q_{\mathrm{n}}$ of the $m$ th and $n$th beads, respectively, and the vacuum dielectric permittivity $\varepsilon_{0}$ are included. A relative dielectric constant of $\varepsilon=15$ is assumed for all electrostatic interactions.

\section{$C G$ parameterization}

The bonded distances and angles for the PGG-PTX-PEGnpRGD molecules were determined as follows: (1) MD simulations of the 12 AA PGG-PTX-PEG-npRGD models were run to $100 \mathrm{~ns}$; (2) the bonded distances and values for the assigned beads were extracted from the AA MD simulations using the AMBER 9.0 ptraj module; and (3) the Boltzmann inversion was used to extract the equilibrium bonded distances and angles [35]. These values were averaged for each set of four PGG-PTX-PEG-npRGD molecules with the same PEG MW. (For example, the bonded distances and angles for the four PEG1000 molecules were averaged together; the resulting values were used for the parameterizing the PGG-PTX-PEG1000npRGD 4 , PGG-PTX-PEG1000-npRGD 8 , PGG-PTXPEG1000-npRGD 12 , and PGG-PTX-PEG1000-npRGD 16 molecules.) $\mathrm{CG}$ topologies for the $\mathrm{GG}, \mathrm{GG}_{\mathrm{COO}-}, \mathrm{GG}_{\mathrm{NH} 3+}$, and GG-PTX residues were taken from Peng et al. and shown in Table S1 [22]; CG topologies for the GGPEG500-npRGD residue are provided in Table S2; and CG topologies of the GG-PEG1000-npRGD and GGPEG2000-npRGD residues are provided in Table S3.

\section{MD simulation}

CG models of the 12 PGG-PTX-PEG-npRGD molecules were constructed using the atom $2 \mathrm{cg}$ and itp-generating scripts provided by the MARTINI tutorials. Each PGGPTX-PEG-npRGD molecule was solvated in explicit solvent comprising of $\mathrm{W}$ beads. Due to the negative charges imparted by the glutamyl-glutamate residues, $234 \mathrm{Na}+$ ions were added in place of water molecules to neutralize the system. The simulations were carried out under NPT (the number of particles $\mathrm{N}$, pressure $\mathrm{P}$, and temperature $\mathrm{T}$ are fixed) conditions at a constant temperature of $310 \mathrm{~K}$ with a coupling constant of $\tau_{\mathrm{T}}=0.1 \mathrm{ps}$ and a constant pressure of 1 bar with a relaxation time of $\tau_{\mathrm{P}}=0.5 \mathrm{ps}$. The cutoff length used for the nonbonded interactions was $r_{\text {cut }}=1.2 \mathrm{~nm}$. Lennard-Jones and Coulombic forces were considered for $r_{\text {cut }}<0.9 \mathrm{~nm}$ and $r_{\text {cut }}<1.2 \mathrm{~nm}$, respectively. Coulombic 
forces were computed every time step for $1.0 \mathrm{~nm}$ and once every 10 time steps for $0.9 \mathrm{~nm}<r_{\text {cut }}<1.2 \mathrm{~nm}$. The time step in the leap-frog integration scheme was $5 \mathrm{fs}$. The energies, coordinates and velocities were written every $0.5 \mathrm{ps}$. MD simulations were run for $1 \mu \mathrm{s}$. The timescale was scaled up by a factor of four because CG dynamics are faster than allatom dynamics, due to the smoothness of the CG interactions. This factor of four is based on the speedup in the diffusional dynamics of CG water as compared to AA water. Therefore, the $1 \mu$ s simulation timescale was scaled up by a factor of four, resulting in an effective time of $4 \mu \mathrm{s}$ $[23,34,36]$.

\section{Root-mean-square deviation clustering}

Root-mean-square deviation (RMSD) clustering - a quantitative method used to analyze protein structures [37] — was used to determine the PGG-PTX-PEG-npRGD conformations. All molecular conformations accessed throughout an MD simulation were categorized into clusters based on structural similarity. For each cluster, a structure from the MD trajectory closest to the conformational average of all structures in the MD trajectory was regarded as the representative conformation. Using the $g_{-}$cluster module of

Table 1 Summary of data from root-mean-square deviation (RMSD) clustering method. The RMSD cutoff lengths optimized for each PGG-PTX-PEG-npRGD molecule and the corresponding number of the GROMACS 4.0.3 package, the RMSD cutoff length was optimized manually so as to meet the following criteria: (1) there were approximately 40 clusters total, (2) very few clusters contained just one representative conformation, and (3) $90 \%$ of the MD trajectory was in clusters fewer than those with one representative conformation. Table 1 provides the optimized RMSD cutoff distances for all 12 PGG-PTXPEG-npRGD molecules. (Sample optimization of the RMSD cutoff length vs. number of clusters generated for the GGPTX-PEG1000-npRGD ${ }_{12}$ molecule is shown in Fig. S2.) After optimization of the RMSD cutoff lengths, the significance of every representative conformation was assessed such that its corresponding frames occupy at least $10 \%$ of the entire $4 \mu$ s trajectory. Once the representative conformations were judged to be sufficiently significant, each was evaluated for its uniqueness as follows: if the RMSD between the significant representative conformations of each significant cluster were greater than twice the RMSD cutoff length, then those significant representative conformation(s) was/were regarded as unique. (For the PGG-PTX-PEG-npRGD molecules with only one significant representative conformation, that conformation was automatically deemed unique.) To verify the uniqueness among clusters, the RMSD Calculator tool in Visual Molecular Dynamics 1.8.6 was used [38].

clusters are listed, as well as the unique and significant central members and the corresponding RMSD between each central member

\begin{tabular}{|c|c|c|c|c|}
\hline System & $\begin{array}{l}\text { RMSD cutoff } \\
(\mathrm{nm})\end{array}$ & $\begin{array}{l}\text { Number of } \\
\text { clusters }\end{array}$ & $\begin{array}{l}\text { Representative } \\
\text { conformations }\end{array}$ & $\begin{array}{l}\text { RMSD between representative } \\
\text { conformations }\end{array}$ \\
\hline
\end{tabular}

\section{PEG500}

4 nonpeptide RGD

8 nonpeptide RGD

12 nonpeptide RGD

16 nonpeptide RGD

0.57
0.59
0.56
0.53

37

38

37

40
A

A

A

A
A
A
B
A
A
B

17.7

7.2

$-$

6.6

\section{PEG1000}

4 nonpeptide RGD

8 nonpeptide RGD

8 nonpeptide RGD

8 nonpeptide RGD

12 nonpeptide RGD

12 nonpeptide RGD

12 nonpeptide RGD

16 nonpeptide RGD
0.59

0.61

0.61

0.61

0.52

0.52

0.52

0.53
37

38

38

38

39

39

39

40

\section{PEG2000}

4 nonpeptide RGD

8 nonpeptide RGD

0.60

12 nonpeptide RGD

0.93

16 nonpeptide RGD

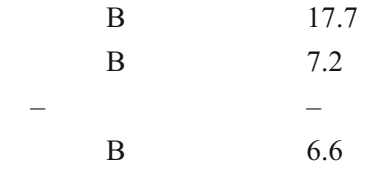

8.0

6.2

4.9

4.1

9.6

7.3

4.3 


\section{Density analysis}

Typically in MD simulations, the radial distribution function, $g(r)$, is used to characterize the structure of the biomolecules. The $g_{\mathrm{AB}}(r)$ between species $\mathrm{A}$ and $\mathrm{B}$ is defined as follows [39]:

$g_{A B}(r)=\frac{\rho_{A B}(r)}{\rho_{B, \text { local }}}$

where $\rho_{\mathrm{AB}}(r)$ is the density of species B at a distance $r$ from species $\mathrm{A}$ and $\rho_{\mathrm{B} \text {,local }}$ is the local density of species $\mathrm{B}$ at maximum distance $r_{\max }$ from species A. For this study, species B refers to a PGG-PTX-PEG-npRGD molecule and species A its center-of-mass (COM). It is important to emphasize that the COM does not necessarily correspond to the geometric center, particularly for irregularly shaped molecules, and the COM is always towards the more massive end.

Since the local densities of the PGG-PTX-PEG-npRGD molecules are different, their $g_{\mathrm{AB}}(r)$ values would not properly describe how the structure of PGG-PTX-PEGnpRGD is affected by PEG MW and npRGD density. Therefore, the density of PGG-PTX-PEG-npRGD from its COM, $\rho_{\mathrm{AB}}(r)$, was used to describe structure. The local density of each PGG-PTX-PEG-npRGD molecule, $\rho_{\mathrm{B}, \text { local }}$, was determined from its molecular mass and simulation box size (see Table 2). The $g$ _rdf module of GROMACS 4.0.3 was then used to extract the $g_{\mathrm{AB}}(r)$ values of each PGGPTX-PEG-npRGD molecule throughout the $4 \mu$ s trajectory. Using the definition of $g_{\mathrm{AB}}(r)$, the density of PGG-PTXPEG-npRGD from its spatial center was determined by multiplying $g_{\mathrm{AB}}(r)$ and $\rho_{\mathrm{B}, \text { local }}: \rho_{\mathrm{AB}}(r)=g_{\mathrm{AB}}(r) \times \rho_{\mathrm{B}, \text { local }}$.

\section{Solvent-accessible surface area analysis}

The solvent-accessible surface area (SASA) indicates the area of a particular species that is exposed to the solvent.
The SASA of the PGG-PTX-PEG-npRGD molecules was analyzed in VMD 1.8.6 using a solvent probe radius of $2.4 \mathrm{~nm}$. For each PGG-PTX-PEG-npRGD molecule, the SASA of the entire molecule was calculated, along with the SASA of PGG, PEG, PTX, and npRGD entities of each PGG-PTX-PEG-npRGD molecule. The contribution of each entity was then determined by taking the fraction of the SASA for a particular entity with respect to the SASA of the whole PGG-PTX-PEG-npRGD molecule, or the percentage (\%) SASA. Table 3 shows the \% SASA for the PGG, PEG, PTX, and npRGD entities for all PGG-PTX-PEG-npRGD molecules. The hydrophilic SASA ( $\left.\% \mathrm{SASA}_{\text {phil }}\right)$ was determined from the sum of the $\% \mathrm{SASA}_{\mathrm{PGG}}$ and $\%$ SASA $_{\text {PEG }}$ residues.

\section{Results and discussion}

Circular dichroism spectroscopy

Circular dichroism spectroscopy was used to determine the secondary structure of a PGG-PTX-PEG220-npRGD 16 molecule. This method of determining the initial structure was originally applied to PGG-PTX in Peng et al. [22]. Although PGG-PTX-PEG220-npRGD 16 was not one of the PGG-PTX-PEG-npRGD molecules pursued in this study, its chemical structure is similar enough to provide an accurate representation of the PGG-PTX-PEG-npRGD molecules being studied.

Figure 4 shows the CD spectra of $1 \mathrm{mg} / \mathrm{ml}$ PGG-PTXPEG220-npRGD ${ }_{16}$ dissolved in $1 \mathrm{X}$ DPBS. The curve is most representative of a random coil at physiological temperature $\left(37^{\circ} \mathrm{C}\right)$. The negative control of $1 \mathrm{X}$ DPBS at $37^{\circ} \mathrm{C}$ indicates that there is no signal interference that may have influenced the conditions while the CD spectrum of PGG-PTX-PEG220-npRGD 16 was being taken. For the
Table 2 Determination of local densities of PGG-PTX-PEGnpRGD molecules. The local density, $\rho_{\mathrm{B} \text {, local }}$, of each PGGPTX-PEG-npRGD molecule was determined by dividing the mass of each molecule by the simulation box volume using the following unit conversions: $6.022 \times 10^{23} \mathrm{Da}=1$ gram and 1 $\mathrm{nm}^{3}=1.0 \times 10^{-21} \mathrm{ml}$

\begin{tabular}{lllll}
\hline System & $\begin{array}{l}\text { Mass } \\
(\mathrm{kDa})\end{array}$ & $\begin{array}{l}\text { Mass } \\
\left(\times 10^{-19} \mathrm{~g}\right)\end{array}$ & $\begin{array}{l}\text { Simulation box } \\
\text { volume }\left(\times 10^{3} \mathrm{~nm}^{3}\right)\end{array}$ & $\begin{array}{l}\text { Local density, } \rho_{\mathrm{B}, \text { local }} \\
\left(\times 10^{-3} \mathrm{~g} / \mathrm{ml}\right)\end{array}$ \\
\hline PGG-PTX-PEG500-npRGD $_{4}$ & 58.6 & 0.97 & 5.60 & 17.4 \\
PGG-PTX-PEG500-npRGD $_{8}$ & 61.9 & 1.03 & 5.07 & 20.3 \\
PGG-PTX-PEG500-npRGD $_{12}$ & 65.1 & 1.08 & 5.72 & 18.9 \\
PGG-PTX-PEG500-npRGD $_{16}$ & 68.5 & 1.14 & 5.54 & 20.5 \\
PGG-PTX-PEG1000-npRGD $_{4}$ & 60.6 & 1.01 & 6.33 & 15.9 \\
PGG-PTX-PEG1000-npRGD $_{8}$ & 65.8 & 1.09 & 6.79 & 16.1 \\
PGG-PTX-PEG1000-npRGD $_{12}$ & 71.0 & 1.18 & 9.35 & 12.6 \\
PGG-PTX-PEG1000-npRGD $_{16}$ & 76.2 & 1.27 & 9.26 & 13.7 \\
PGG-PTX-PEG2000-npRGD $_{4}$ & 64.8 & 1.08 & 13.4 & 8.03 \\
PGG-PTX-PEG2000-npRGD $_{8}$ & 74.2 & 1.23 & 16.3 & 7.58 \\
PGG-PTX-PEG2000-npRGD $_{12}$ & 83.7 & 1.39 & 24.1 & 6.34 \\
PGG-PTX-PEG2000-npRGD $_{16}$ & 93.1 & 1.55 & 24.4 & \\
\hline
\end{tabular}


Table 3 Percentage solventaccessible surface area (SASA) of PGG-PTX-PEG-npRGD molecules. Lists the percentage SASA values for the PGG, PEG, PTX, and npRGD entities with respect to the entire SASA of each PGG-PTX-PEG-npRGD molecule

*The $\% \mathrm{SASA}_{\text {phil }}$ is the sum of the $\% \mathrm{SASA}_{\mathrm{PGG}}$ and $\% \mathrm{SASA}_{\mathrm{PEG}}$

\begin{tabular}{|c|c|c|c|c|c|}
\hline System & $\% \mathrm{SASA}_{\mathrm{PGG}}$ & $\% \mathrm{SASA}_{\mathrm{PEG}}$ & $\% \mathrm{SASA}_{\mathrm{phil}} *$ & $\% \mathrm{SASA}_{\mathrm{PTX}}$ & $\% \mathrm{SASA}_{\mathrm{npRGD}}$ \\
\hline \multicolumn{6}{|l|}{ PEG 500} \\
\hline \multicolumn{6}{|l|}{4 nonpeptide RGD } \\
\hline A & 78.5 & 1.1 & $79.6^{*}$ & 18.2 & 2.2 \\
\hline B & 69.5 & 1.9 & $71.4^{*}$ & 25.7 & 2.9 \\
\hline \multicolumn{6}{|l|}{8 nonpeptide RGD } \\
\hline A & 73.1 & 1.6 & $74.7 *$ & 21.5 & 3.9 \\
\hline $\mathrm{B}$ & 73.3 & 1.5 & $74.8^{*}$ & 20.4 & 4.9 \\
\hline 12 nonpeptide RGD & 68.2 & 2.8 & $70.0^{*}$ & 19.0 & 8.2 \\
\hline \multicolumn{6}{|l|}{16 nonpeptide RGD } \\
\hline A & 66.9 & 3.7 & $70.6^{*}$ & 18.7 & 10.6 \\
\hline $\mathrm{B}$ & 58.1 & 6.2 & $64.3^{*}$ & 23.8 & 11.8 \\
\hline \multicolumn{6}{|l|}{ PEG1000 } \\
\hline \multicolumn{6}{|l|}{4 nonpeptide RGD } \\
\hline A & 74.2 & 0.3 & $74.5^{*}$ & 24.2 & 1.2 \\
\hline $\mathrm{B}$ & 74.9 & 1.1 & $76.0^{*}$ & 22.3 & 1.8 \\
\hline \multicolumn{6}{|l|}{8 nonpeptide RGD } \\
\hline A & 78.3 & 2.0 & $80.3 *$ & 16.4 & 3.4 \\
\hline B & 64.7 & 5.8 & $70.5^{*}$ & 25.2 & 4.3 \\
\hline $\mathrm{C}$ & 71.7 & 2.4 & $74.1^{*}$ & 22.8 & 3.1 \\
\hline \multicolumn{6}{|l|}{12 nonpeptide RGD } \\
\hline A & 78.7 & 0.5 & $79.2^{*}$ & 15.0 & 5.8 \\
\hline $\mathrm{B}$ & 73.2 & 0.4 & $73.6^{*}$ & 18.7 & 7.7 \\
\hline $\mathrm{C}$ & 73.9 & 0.4 & $74.3^{*}$ & 18.8 & 6.9 \\
\hline 16 nonpeptide RGD & 74.1 & 0.6 & $74.7^{*}$ & 16.0 & 9.2 \\
\hline \multicolumn{6}{|l|}{ PEG2000 } \\
\hline 4 nonpeptide RGD & 78.9 & 1.8 & $80.7 *$ & 17.8 & 1.5 \\
\hline 8 nonpeptide RGD & 72.6 & 6.5 & $79.1^{*}$ & 17.0 & 3.8 \\
\hline 12 nonpeptide RGD & 68.3 & 8.4 & $76.7^{*}$ & 16.9 & 6.4 \\
\hline 16 nonpeptide RGD & 66.0 & 12.5 & $78.5^{*}$ & 12.3 & 9.2 \\
\hline
\end{tabular}

positive control, the shape of the alpha-helical FD protein, a bZIP transcription factor in a floral pathway [40], in 50\% $\mathrm{TFE} / 50 \% \mathrm{ddH}_{2} \mathrm{O} / 0.1 \%$ TFA buffer does indeed correspond to an alpha-helix, as compared with the CD spectrum of the

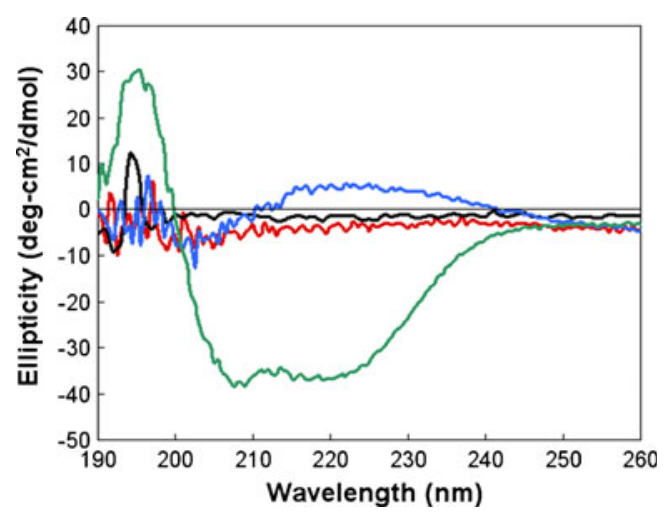

Fig. 4 Circular dichroism spectra of samples. The spectra for 16 nonpeptide RGD-targeted PGG-PTX (red), PGG-PTX (blue), FD protein (green), and pure 1X DPBS (black) are shown alpha-helical poly $(\gamma$-tyrosine) [41]. The lowest part of the curve is $\sim 215 \mathrm{~nm}$ and the highest point of the curve is $\sim 200 \mathrm{~nm}$, collectively indicating that the FD protein is alpha-helical. Since PGG-PTX-PEG220-npRGD ${ }_{16}$ exists as a random coil, this data suggest that the PGG-PTX-PEGnpRGD molecules being modeled most likely exist as random coils. Therefore, their existence as random coils imposes no stipulations on the construction of AA models of PGG-PTX-PEG-npRGD. Also, the CD spectroscopy results further support the theory that the conformation of PGG-PTX-PEG-npRGD is not limited to a specific structure, and structural analysis must account for multiple conformations of PGG-PTX-PEG-npRGD.

RMSD values

Figure 5 shows the RMSD time evolutions for all PGG-PTX-PEG-npRGD molecules. Most time evolutions increased up to $\sim 6-7 \mathrm{~nm}$ and reached a statistical equilibrium by $4 \mu \mathrm{s}$. Given the relatively large sizes of 

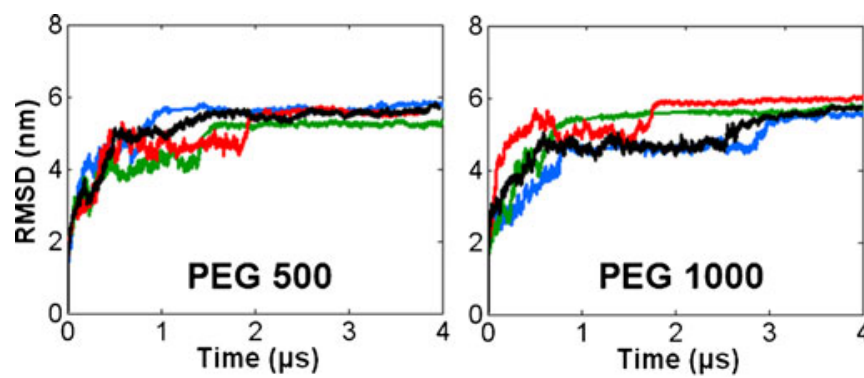

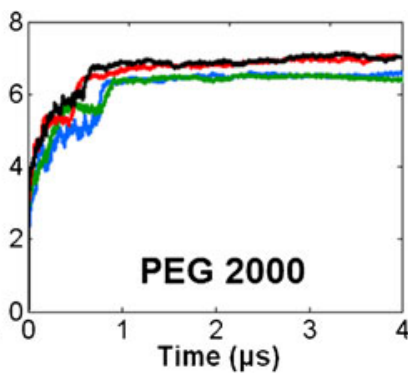

Fig. 5 RMSD time evolutions of PGG-PTX-PEG-npRGD molecules. Data for molecules with 4 (PEG) $)_{n}$-nonpeptide RGD molecules (blue), 8 (PEG) ${ }_{n}$-nonpeptide RGD molecules (green), 12 (PEG) $)_{n}$-nonpeptide

these systems, these values are reasonable. The RMSD values for the PEG2000 molecules are slightly higher than those of the PEG500 and PEG1000 molecules (by $\sim 1 \mathrm{~nm}$ ), whereas the RMSD values for the PEG500 and PEG1000 molecules are comparable. This $1 \mathrm{~nm}$ difference is in accordance with the size of the molecular systems, as the PEG2000 molecules are the largest. In addition, the time it takes for PGG-PTX-PEG-npRGD molecules to attain statistical equilibrium depends on the PEG MW. The PEG1000 molecules take the longest time to reach equilibrium, as some molecules do not reached a plateau until $3 \mu$ s. Conversely, the PEG2000 molecules take the least time to reach equilibrium; by $1 \mu \mathrm{s}$, all molecules have attained a statistical equilibrium. The time it takes for the PEG500 molecules to level off is $\sim 2 \mu \mathrm{s}$, which is intermediate compared to the time it takes for the PEG1000 and PEG2000 molecules to reach equilibrium. This behavior suggests that the PEG1000 molecules fluctuate the most, accessing a wide range of molecular conformations throughout the $4 \mu$ s MD trajectory.

\section{Size of a PGG-PTX-PEG-npRGD molecule}

Figure 6 shows the radius of gyration $\left(R_{\mathrm{gyr}}\right)$ trajectories for all PGG-PTX-PEG-npRGD molecules over the $4 \mu$ s time scale. For all molecules, the $R_{\text {gyr }}$ values begin at $\sim 7-8 \mathrm{~nm}$ and decrease to $\sim 3 \mathrm{~nm}$, nearly half their original size. The PEG2000 and PEG1000 systems take the shortest and
RGD molecules (red), and 16 (PEG) $)_{n}$-nonpeptide RGD molecules (black) are shown

longest to reach their final $R_{\mathrm{gyr}}$ values, respectively. These values correspond to the RMSD values: the PEG2000 and PEG1000 systems also take the shortest and longest to reach their final RMSD values, respectively. Furthermore, as shown in Fig. 7, the $R_{\text {gyr }}$ of each PGG-PTX-PEGnpRGD representative conformation is approximately 2$4 \mathrm{~nm}$. Figure 8 further supports the notion that neither the PEG MW nor the npRGD density significantly influences the size of a PGG-PTX-PEG-npRGD molecule.

Structure of a PGG-PTX-PEG-npRGD molecule

Figure 9 shows that each PGG-PTX-PEG-npRGD molecule is most densely packed at its COM. Most PGG-PTX-PEGnpRGD molecules exhibit a peak density of $\sim 1 \mathrm{~g} / \mathrm{ml}$ just outside its center-of-mass at $r \sim 1 \mathrm{~nm}, 1.5 \mathrm{~nm}$, and $2 \mathrm{~nm}$ for the PEG500, PEG1000, and PEG2000 molecules. This increase in distance of peak density is correlated with increasing PEG MW. Figure 8 shows how the density of each residue type (PGG, PTX, PEG, and npRGD) varies from the COM of a PGG-PTX-PEG-npRGD molecule. The PEG MW impacts how the density of each residue changes with distance from COM. For instance, PTX and npRGD residues exhibit peak densities at greater distances as PEG MW increases, suggesting that higher PEG MW causes PTX and npRGD residues to approach closer to the surface of the PGG-PTX-PEG-npRGD. The peak densities of PTX
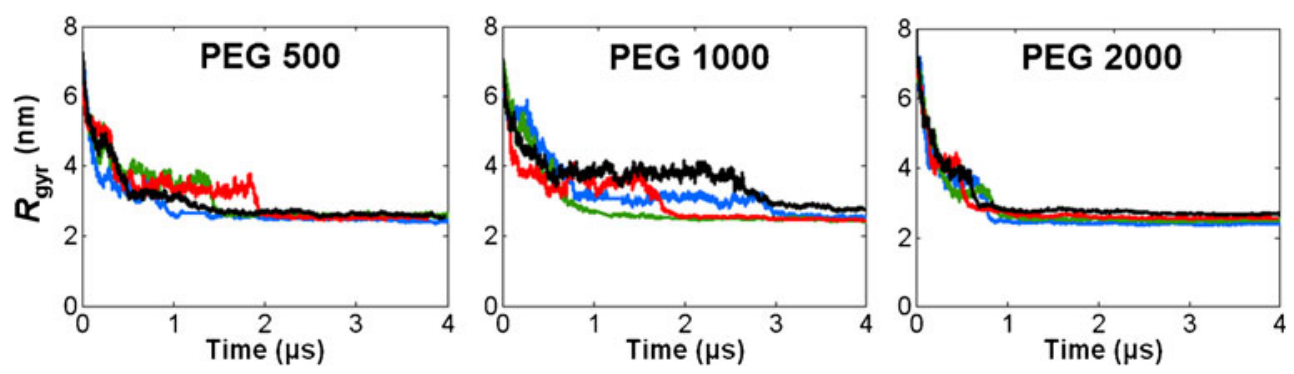

Fig. 6 Radius of gyration trajectories of PGG-PTX-PEG-npRGD PTX molecules. Data for molecules with $4(\mathrm{PEG})_{\mathrm{n}}$-nonpeptide RGD molecules (blue), 8 (PEG) $)_{\mathrm{n}}$-nonpeptide RGD molecules (green), 12
$(\mathrm{PEG})_{\mathrm{n}}$-nonpeptide RGD molecules (red), and 16 (PEG) $)_{\mathrm{n}}$-nonpeptide RGD (black) molecules are shown 


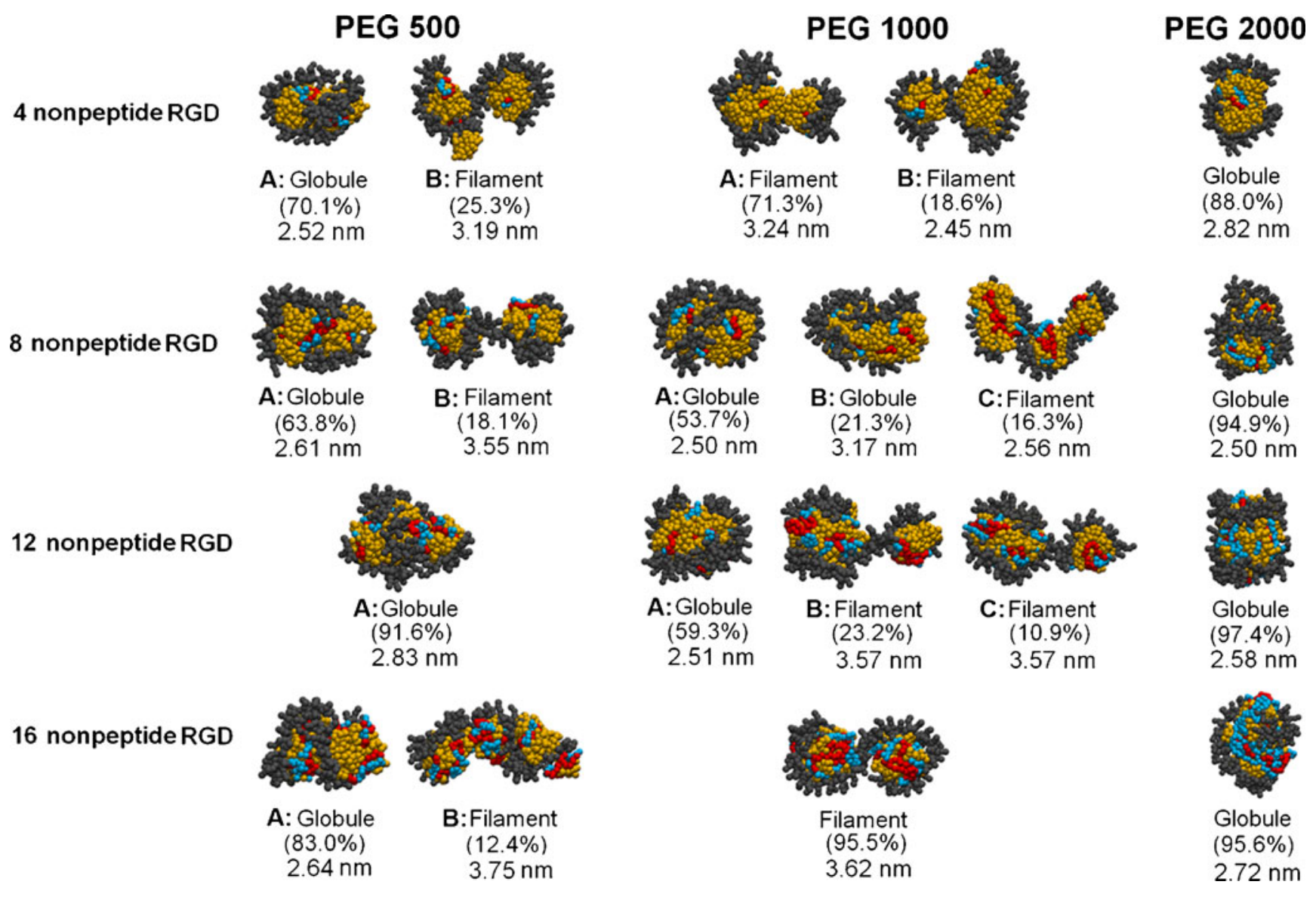

Fig. 7 Representative conformations of PGG-PTX-PEG-npRGD molecules. Each structure shows GG (grey), PTX (yellow), PEG (blue), and npRGD (red) residues. Explicit W beads and $\mathrm{Na}^{+}$ions are not shown. Representing an actual frame in the trajectory, the central member embodies the configuration that is the most similar to the

and npRGD in Fig. 8 agree with the peak densities of PGGPTX-PEG-npRGD in Fig. 9, suggesting that the presence of PTX and npRGD residues is mostly responsible for this spatial shift in the peak density of the overall PGG-PTXPEG-npRGD molecule. On the contrary, for PEG, the distance of peak density tends to decrease as the PEG MW increases, which suggests that increasing the PEG spacer length causes PEG to shift away from the external surface and move internally towards the COM of a PGG-PTXPEG-npRGD molecule. In general, the difference in peak densities of PEG and peak densities of PTX and npRGD becomes more pronounced as the PEG MW increases, which signifies that the PGG-PTX-PEG-npRGD molecule tends to become more spatially divided (by residue type) at higher PEG MW. Finally, taking the $R_{\mathrm{gyr}}$ values into account, these results suggest that while the PEG MW does not affect the size of a PGG-PTX-PEG-npRGD molecule, the PEG MW does impact structure. Figure 8 also suggests that the npRGD density also does not significantly affect the structure of the PGG-PTX-PEG-npRGD molecule. average of all configurations in that cluster. The percentage indicates the population of frames, or $\%$ trajectory occupancy, corresponding to that particular cluster for the full $4 \mu \mathrm{s}$ MD trajectory. Radius of gyration values for each PGG-PTX-PEG-npRGD molecule is also shown below the $\%$ trajectory occupancy

Implications of shape of a PGG-PTX-PEG-npRGD molecule

Discher and colleagues have demonstrated that filamentous particles retains a longer circulation half-life in the bloodstream, thus decreasing levels of clearance via the reticoendothelial system (RES) and improving the chances of the nanoparticle to reach the target tumor [16]. Also, nonspherical nanoparticles have been shown to adhere more firmly to walls of tumor endothelia [17]. Figure 7 shows the representative conformations of all PGG-PTXPEG-npRGD molecules. The geometry of each molecule is characterized as a filament (wormlike and threadlike) or globule (round). In general, most molecules are globules, which is most likely attributed to the hydrophobic driving force among PTX molecules that are uniformly distributed along the PGG backbone. The uniform distribution of PTX causes the entire PGG-PTX-PEG-npRGD to self-assemble into a globule in which PTX molecules are located in the core, where it is the furthest from the solvent and surrounded by hydrophilic PGG. Other molecules, such as 

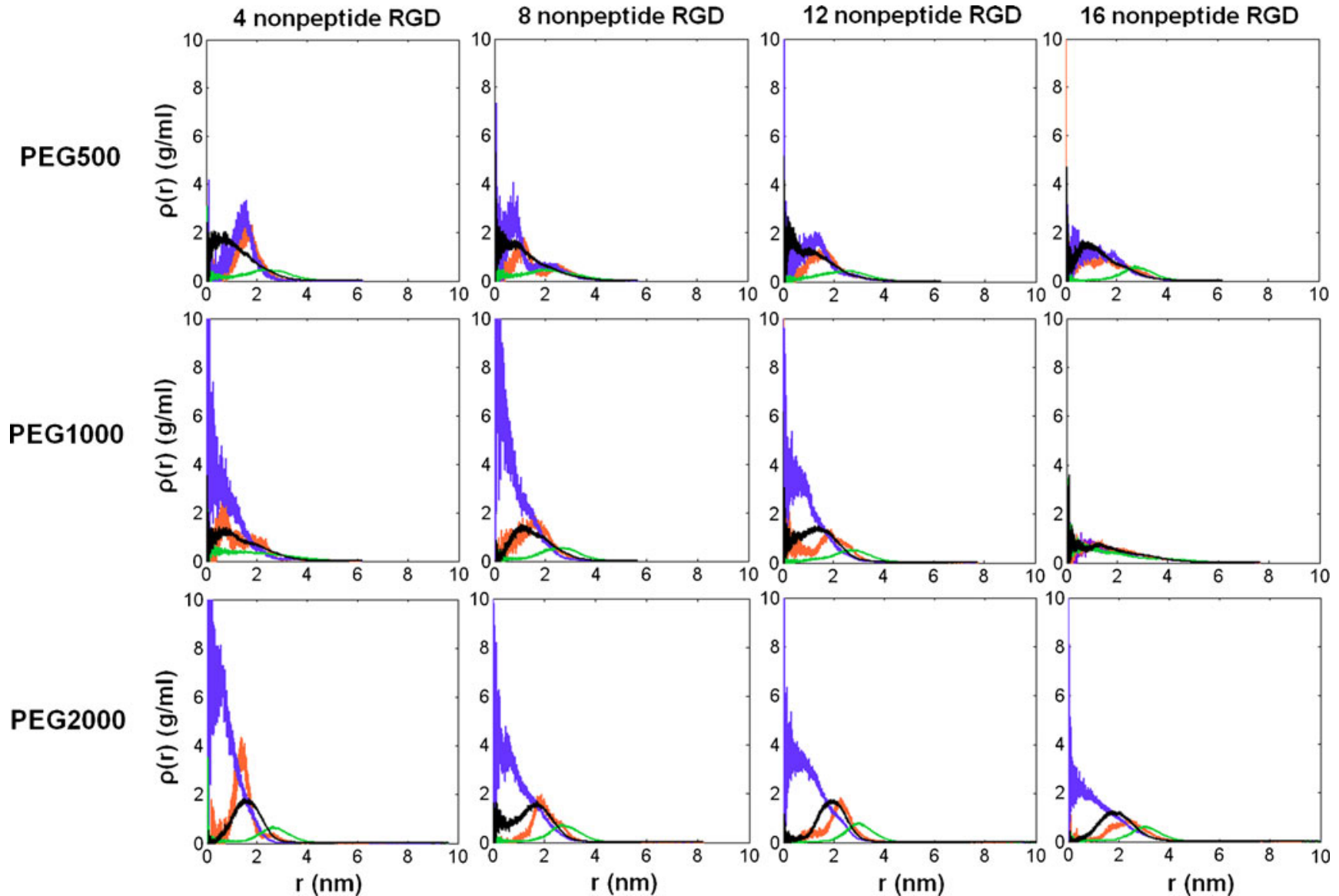

Fig. 8 Density of residues from COM of a PGG-PTX-PEG-npRGD molecules. Data for poly(glutamyl-glutamate) (green), paclitaxel (black), poly(ethylene) glycol (purple), and nonpeptide RGD (orange) are shown

PGG-PTX-PEG1000-npRGD 4 and PGG-PTX-PEG1000$n p \mathrm{RD}_{12}$, exhibit both filamentous and globular behavior. The shape of the PGG-PTX-PEG-npRGD molecule is not significantly affected by the npRGD density but by the PEG MW. The PEG1000 molecules exhibit the most filamentous behavior, as each one of its four molecules is characterized by a filamentous conformation. The higher prominence of filaments in the PEG500 and PEG1000 molecules is possibly a result of the sterical interactions among the rigid, conformationally constrained nonpeptide RGD residues that hinder the formation of a more compact globule. For the PEG2000 molecules, however, the prominence of the globular shapes ( 90\%) suggests that 2,000 $\mathrm{Da}$ is the PEG MW most likely to prevent sterical interactions among nonpeptide RGD residues, thus allowing the PGG-PTX-PEG-npRGD molecules to form a globule. Overall, the results suggest that PGG-PTX-PEGnpRGD molecules with PEG500 and PEG1000 spacers are
Fig. 9 Density of PGG-PTXPEG-npRGD molecules from COM. Data for molecules with 4 $(\mathrm{PEG})_{\mathrm{n}}$-nonpeptide RGD molecules (blue), 8 (PEG) $)_{\mathrm{n}}$-nonpeptide RGD molecules (green), 12 $(\mathrm{PEG})_{\mathrm{n}}$-nonpeptide RGD molecules $(r e d)$, and 16 (PEG) $)_{\mathrm{n}}$-nonpeptide RGD molecules (black) are shown
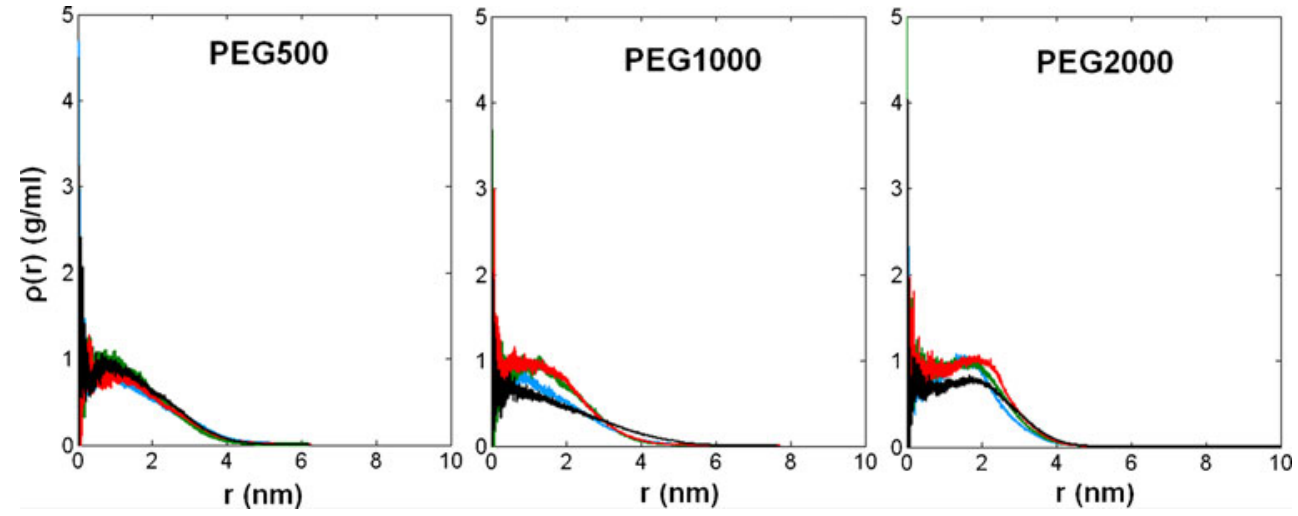
more efficacious than the PGG-PTX-PEG-npRGD molecules with PEG2000 spacers. While the molecular weight of the PEG spacer influences the shape of a PGG-PTX-PEG-npRGD molecule, the npRGD density has only minimal impact.

\section{Implications of flexibility of a PGG-PTX-PEG-npRGD molecule}

Takeoka has speculated that higher molecular flexibility of nanoparticles promotes more surface interactions to tumor cells [18]. The flexibility of each PGG-PTX-PEG-npRGD molecule was assessed by the number of representative conformations of each molecule and, for those systems with multiple representative conformations, the similarity among representative conformations. A higher number of representative conformations indicates a stronger tendency for that molecule to fluctuate and change shape. The PEG1000 molecules have the most representative conformations; the PGG-PTX-PEG1000-npRGD 8 and PGG-PTX-PEG1000$n p R G D_{12}$ molecules are characterized by three representative conformations, although with low significance $(\sim 10$ $20 \%$ ). On the contrary, each PEG2000 molecule has only one unique shape with very high significance $(\sim 90 \%)$. Moreover, the degree of similarity among the representative conformations is also an indicator of flexibility: a PGGPTX-PEG-npRGD molecule with two globular representative conformations is less flexible than a molecule characterized by globular and filamentous conformations. For instance, PGG-PTX-PEG500-npRGD 4 and PGG-PTXPEG1000-npRGD 4 each has two representative conformations, but a larger structural difference exists between the globular and filamentous shapes of the PGG-PTX-PEG500$\mathrm{npRGD}_{4}$ molecule, as compared to the two filamentous conformations of the PGG-PTX-PEG1000-npRGD 4 molecule. In all, these observations show that the PEG2000 molecules are the least flexible, whereas the PEG500 and PEG1000 molecules are more flexible and would therefore bind more effectively to tumor cells. Finally, the npRGD ligand density does not have a significant effect on the flexibility of a PGG-PTX-PEG-npRGD molecule.

Implications of surface hydrophilicity of a PGG-PTX-PEGnpRGD molecule

Nanoparticles with higher degrees of surface hydrophilicity have been shown to possess longer circulation half-lives in the bloodstream, suggesting a higher preference for accumulating in leaky vasculature of tumors [14]. The degree of surface hydrophilicity of each PGG-PTX-PEGnpRGD molecule was assessed by the \% SASA of the hydrophilic entities: PGG and PEG (see Table 3). These \% SASA values are related to the density plots in Fig. 9. In general, the high $\% \mathrm{SASA}_{\mathrm{PGG}}$ values can be attributed to the high density values at $r \sim 3 \mathrm{~nm}$, at the external surface of the PGG-PTX-PEG-npRGD molecule. Likewise, the \%

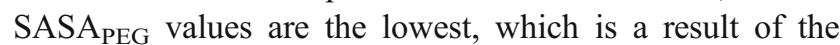
high concentration of PEG near the center-of-mass and not the surface. Figure 8 also shows that the densities of PTX and npRGD residues are relatively high near the surface of a PGG-PTX-PEG-npRGD molecule, but their low molecular weight ratio with respect to the entire molecule results in lower $\% \mathrm{SASA}_{\mathrm{PTX}}$ and $\% \mathrm{SASA}_{\text {nPRGD }}$ values than $\%$ $\mathrm{SASA}_{\mathrm{PGG}}$. As expected, the surface hydrophilicity increases with PEG $\mathrm{MW}$, and an increase in npRGD density results in higher $\% \mathrm{SASA}_{\text {npRGD. }}$. A higher npRGD density also tends to cause a decrease in the $\% \mathrm{SASA}_{\text {phil }}$. While nonpeptide RGD is used to promote active targeting of PGG-PTX to tumor cells, too many nonpeptide RGD ligands may decrease surface hydrophilicity, which may unfavorably decrease to the circulation half-life. PGGPTX-PEG-npRGD must remain in the bloodstream long enough to diffuse convectively into leaky tumor vasculature. At the same time, adequate amounts of nonpeptide RGD ligands must be exposed to the solvent to achieve effective binding to $\alpha_{V} \beta_{3}$ integrins. Therefore, optimization of npRGD density is necessary to attain optimal efficacy of PGG-PTX-PEG-npRGD. Taking these factors into consideration, the PGG-PTX-PEG-npRGD molecules with 8 and 12 npRGD and PEG1000 and PEG2000 MW are most likely to confer the longest circulation half-lives and effective targeting to $\alpha_{\mathrm{V}} \beta_{3}$ integrins on tumor cells.

\section{Recommendations}

This study was founded on the hypothesis that the degree of PEGylation (500 Da; 1,000 Da; and 2,000 Da) and npRGD density $(4,8,12$, and 16 nonpeptide RGD per PGG-PTXPEG-npRGD molecule) collectively affect the size, structure, shape, flexibility, and surface hydrophilicity of PGG-PTXPEG-npRGD. While the size of a PGG-PTX-PEG-npRGD molecule is consistent at all PEG MW and npRGD densities, the molecular density tends to be is higher at the surface at higher PEG MW. The results also show that the PEG500 and PEG1000 molecules exhibited more filamentous behavior and higher flexibility, whereas the PEG2000 molecules are globular and more rigid. An increase in the nonpeptide RGD ligand density may result in lower surface hydrophilicity, yet an insufficient number of nonpeptide RGD ligands may prevent PGG-PTX-PEG-npRGD from achieving effective targeting to tumor cells. All factors considered, the ideal PGG-PTX-PEG-npRGD candidate would possess the following properties: filamentous shape, high flexibility, and high surface hydrophilicity. Based on the results, the PGG-PTX-PEG1000-npRGD 4 and PGG-PTXPEG1000-npRGD 8 molecules most satisfy these criteria and are the most promising candidates. 


\section{Conclusions}

The roles of shape, flexibility, and surface hydrophilicity in influencing the delivery of therapeutic nanoparticles to tumors have attracted increasing attention. These factors can serve as a basis for the design of anticancer therapeutics, such as PGG-PTX-PEG-npRGD. While it may be technically feasible to determine these properties using experimental methods, the effort required to synthesize all candidate compounds and subsequently perform experimental testing would be very time- and resourceintensive, especially since the testing is usually conducted in a trial-and-error manner. Therefore, MD simulations were used to explore the size, shape, flexibility, and surface hydrophilicity of candidate PGG-PTX-PEGnpRGD compounds in a more efficient and controlled manner. The theoretical results can be used to further guide experiments, potentially shortening the preclinical development of PGG-PTX-PEG-npRGD. This study also introduces a new structural analysis method of characterizing semiflexible molecules without a specific, crystallized conformation.

Acknowledgments Financial support for this work was provided by a University of California Discovery Grant and the Nitto Denko Technical Corporation (NDT). S.K.D. and L.Y. are full-time employees of NDT, and S.B.H. and D.A.G. serve as consultants for NDT on an arrangement approved by the UCSD Conflict of Interest Committee. The authors acknowledge J. Andrew McCammon for providing the supercomputing cluster resources.

Open Access This article is distributed under the terms of the Creative Commons Attribution Noncommercial License which permits any noncommercial use, distribution, and reproduction in any medium, provided the original author(s) and source are credited.

\section{References}

1. Ferrari M (2005) Cancer nanotechnology: opportunities and challenges. Nat Rev Cancer 5:161-171

2. Amiji MM (2007) Nanotechnology for cancer therapy. CRC, Boca Raton

3. Satchi-Fainaro R, Puder M, Davies JW, Tran HT, Sampson DA, Greene AK, Corfas G, Folkman J (2004) Targeting angiogenesis with a conjugate of HPMA copolymer and TNP-470. Nat Med 10:225-261

4. Kok RJ, Schraa AJ, Bos EJ, Moorlag HE, Asgeirsdottir SA, Everts M, Meijer DKF, Molema G (2002) Preparation and functional evaluation of RGD-modified proteins as $\alpha \mathrm{V} \beta 3$ integrin directed therapeutics. Bioconjug Chem 13:128-135

5. Chen X, Plasencia C, Hou Y, Neamati N (2005) Synthesis and biological evaluation of dimeric RGD peptide-paclitaxel conjugate as a model for integrin-targeted drug delivery. J Med Chem 48:1098-1106

6. Lorger M, Kreuger JS, O'Neal M, Staflin K, Felding-Habermann B (2009) Activation of tumor cell integrin $\alpha \mathrm{V} \beta 3$ controls angiogenesis and metastatic growth in the brain. Proc Natl Acad Sci USA 106:10666-10671

7. Temming K, Schiffelers RM, Molem G, Kok RJ (2005) RGDbased strategies for selective delivery of therapeutics and imaging agents to the tumour vasculature. Drug Resist Updat 8:381-402

8. Danhier F, Vroman B, Lecouturier N, Crokart N, Pourcelle V, Freichels $\mathrm{H}$, Jérôme $\mathrm{C}$, Marchand-Brynaert J, Feron $\mathrm{O}$, Préat $\mathrm{V}$ (2009) Targeting of tumor endothelium by RGD-grafted PLGAnanoparticles loaded with paclitaxel. J Control Release 2:166-173

9. Bourguet E, Banères JL, Parello J, Lusinchi X, Girard JP, Vidal JP (2003) Nonpeptide RGD antagonists: a novel class of mimetics, the 5, 8-disubstituted 1-azabicyclo[5.2.0]nonan-2-one Lactam. Bioorg Med Chem Lett 13:1561-1564

10. Wang X, Gang Z, Van S, Yu L (2009) Polymer paclitaxel conjugates and methods for treating cancer. USA Patent US20090226393

11. Pujol JL (2005) Phase II study of carboplatin and weekly paclitaxel as first line treatment of elderly patients with non-small cell lung cancer. Clinical Study Report for Study CA139-368

12. Yoshida T (2009) Rollover study of weekly paclitaxel (BMS181339) in patients with advanced breast cancer. Clinical Study Report for Study CA139-387

13. Duncan R (2003) The dawning era of polymer therapeutics. Nat Rev Drug Discov 2:347-360

14. McNeeley KM, Karathanasis E, Annapragada AV, Bellamkonda RV (2009) Masking and triggered unmasking of targeting ligands on nanocarriers to improve drug delivery to brain tumors. Biomaterials 30:3986-3995

15. Farokhzad OC, Langer R (2009) Impact of nanotechnology on drug delivery. ACS Nano 3:16-20

16. Geng Y, Dalhaimer P, Cai S, Tsai R, Tewari M, Minko T, Discher DE (2007) Shape effects of filaments versus spherical particles in flow and drug delivery. Nat Nanotechnol 2:249-255

17. Decuzzi P, Pasqualini R, Arap W, Ferrari M (2009) Intravascular delivery of particulate systems: does geometry really matter? Pharma Res 26:235-243

18. Takeoka S (2002) Rolling properties of rGPIb $\alpha$-conjugated phospholipid vesicles with different membrane flexibilities on vWf surface under flow conditions. Biochem Biophys Res Commun 296:765-770

19. van Gunsteren WF (1993) Molecular dynamics studies of proteins. Curr Opin Struct Biol 3:277-281

20. Mark AE, Berendsen HJC, van Gunsteren WF (1991) Conformational flexibility of aqueuous monomeric and dimeric insulin: a molecular dynamics study. Biochemistry 30:10866-10872

21. Daggett V, Levitt M (1992) A model of the molten globule state from molecular dynamics simulations. Proc Natl Acad Sci USA 89:5142-5146

22. Peng LX, Ivetac A, Van S, Zhao G, Chaudhari AS, Yu L, Howell SB, McCammon JA, Gough DA (2010) Characterization of a clinical polymer-drug conjugate using multiscale modeling. Biopolymers 93:936-951

23. Monticelli L, Kandasamy SK, Periole X, Larson RG, Tieleman DP, Marrink SJ (2008) The MARTINI coarse-grained force field: extension to proteins. J Chem Theory Comput 4:819-834

24. Greenfield NJ (2007) Using circular dichroism spectroscopy spectra to estimate protein secondary structure. Nat Protoc 1:2876-2890

25. Bernstein FC, Koetzle TF, Williams GJ, Meyer EE Jr, Brice MD, Rodgers JR, Kennard O, Shimanouchi T, Tasumi M (1977) J Mol Biol 112:535

26. Frisch MJT et al (1995) Gaussian 94. Gaussian Inc, Pittsburgh

27. Cornell WD, Cieplak C, Bayly CI, Kollman PA (1993) Application of RESP charges to calculate conformational energies, 
hydrogen bond energies, and free energies of solvation. J Am Chem Soc 115:9620-9631

28. Onufriev A, Bashford D, Case DA (2004) Exploring protein native states and large-scale conformational changes with a modified Generalized-Born model. Proteins 55:383-394

29. Weiser J, Shenkin PS, Still WC (1999) Approximate atomic surfaces from linear combinations of pairwise overlaps (LPCO). J Comput Chem 20:217-230

30. Hornak V, Abel R, Okur A, Strockbine R, Roitberg A, Simmerling $C$ (2006) Comparison of multiple Amber force fields and development of improved protein backbone parameters. Proteins 65:712-725

31. Cornell WD, Cieplak P, Bayly CI, Gould IR, Merz KM Jr, Ferguson DM, Spellmeyer DC, Fox T, Caldwell JW, Kollman PA (1995) A second generation force field for the simulation of proteins, nucleic acids, and organic molecules. J Am Chem Soc 117:5179-5197

32. Wang J, Rolf RM, Caldwell JW, Kollman PA, Case DA (2004) Development and testing of a general amber force field. J Comput Chem 25:1157-1174

33. Ryckaert J, Ciccotti G, Berendsen H (1977) Numerical integration of the Cartesian equations of motion of a system with constraints; molecular dynamics of n-alkenes. J Comp Physiol 23:327-341
34. Marrink SJ, Rissalada JH, Yefimov S, Tieleman DP, de Vries AH (2007) The MARTINI force field: coarse-grained model for biomolecular simulations. J Phys Chem B 111:7812-7824

35. Trylska J, Tozzini V, McCammon JA (2005) Exploring global motions and correlations in the ribosome. Biophys $\mathrm{J}$ 89:14551463

36. Marrink SJ, de Vries AH, Mark AE (2004) J Phys Chem B 108:750-760

37. Cheng LS, Amaro RE, Xu D, Li WW, Arzberger PW, McCammon JA (2008) Ensemble-based virtual screening reveals potential novel antiviral compounds for avian influenza neuraminidase. J Med Chem 51:3878-3894

38. Humphrey W, Dalke A, Schulten K (1996) VMD - Visual Molecular Dynamics. J Mol Graph 14:33-38

39. Lindahl E, Hess B, van der Spoel D (2001) GROMACS 3.0: a package for molecular simulation and trajectory analysis. J Mol Model 7:306-317

40. Abe M, Kobayashi Y, Yamamoto S, Daimon Y, Yamaguchi A, Ikeda Y, Ichinoki H, Notaguchi M, Goto K, Araki T (2005) FD, a bZIP protein mediating signals from the floral pathway integrator FT at the shoot apex. Science 309:1052-1056

41. Yasui SC, Keiderling TA (1986) Vibrational circular dichroism of polypeptides. VI. Polytyrosine alpha-helical and random-coil results. Biopolymers 25:5-15 